hep-th/0312076

\title{
On effective action of string theory flux compactifications
}

\author{
Alex Buchel \\ Department of Applied Mathematics \\ University of Western Ontario \\ London, Ontario N6A 5B7, Canada \\ Perimeter Institute for Theoretical Physics \\ Waterloo, Ontario N2J 2W9, Canada
}

\begin{abstract}
We discuss four dimensional effective actions of string theory flux compactifications. These effective actions describe four dimensional gravity coupled to overall Kähler modulus of the compactification manifold. We demonstrate the agreement between ten dimensional equations of motion of supergravity with localized branes, and equations of motion derived from the effective action. The agreement is lost however if one evaluates the full effective action on the equations of motion for a subset of the supergravity modes, provided these modes depend on-shell on the Kähler modulus.
\end{abstract}

December 2003 


\section{Introduction}

Probably the biggest surprise of recent cosmological measurements is the evidence that the cosmological constant of the Universe is positive. It is thus important to understand how (and if) four dimensional de-Sitter vacua arise in string theory compactifications. The problem is complicated by the well known no-go theorem in supergravity $[1,2]$ which forbids non-singular (warped) de-Sitter compactifications with finite four dimensional Newton's constant ${ }^{1}$. This no-go theorem can be phrased in the statement on the energy condition on the matter stress tensor upon consistent Kaluza-Klein (KK) reductions $^{2}$ : if the matter of the higher dimensional theory obeys the strong energy condition, the matter of the lower dimensional effective description would satisfy the strong energy condition as well, implying that lower dimensional de Sitter (or more generally accelerating Universe) is not possible. In ten dimensional supergravities matter satisfies the strong energy condition.

The situation is different with positive tension extended objects, D-branes, in string theory. In ten dimensions, whether or not a $\mathrm{D} p$-brane satisfies the strong energy condition depends on its codimension. Specifically, codimension three and higher branes $(p<7)$ satisfy strong energy condition. In consistent supergravity KK reductions the effective lower dimensional action can be obtained by integrating out compact directions of a ten dimensional supergravity effective action. If one assumes that the same prescription is true for a D-brane effective action, one immediately concludes that upon KK reduction D-branes can be sources for de Sitter. The point simply is that KK reduction transverse to a brane decreases its codimension, thus making lower dimensional accelerating Universe possible. In explicit string construction this idea has been realized for the first time in [8], KKLT. The starting point of KKLT construction is type IIB string theory compactified on Calabi-Yau (CY) 3 -folds $\mathcal{M}_{6}$ with imaginary self-dual (ISD) 3-form fluxes. Classically, as discussed in details in [9,10] (GKP), for a suitable choice of a CY and fluxes, one can fix all complex structure moduli (including the axion-dilaton), and get in four dimensions no-scale models [11-13]: a vanishing four dimensional cosmological constant and a complex Kähler modulus $\rho$ (related to the overall size of the compactification manifold). This no-scale structure is expected

\footnotetext{
${ }^{1}$ Having a finite four dimensional Newton's constant is a crucial qualifier, as it is straightforward to construct explicit supergravity warped product backgrounds $d S_{4} \times \mathcal{M}_{6}$ for non-compact six-dimensional manifolds $\mathcal{M}_{6}[3-5]$.

${ }^{2}$ Most recently this has been emphasized in $[6,7]$.
} 
to be spoiled by string tree level $\alpha^{\prime}$ corrections, as well as perturbative string loops and string instanton corrections [10]. Indeed, in KKLT it was demonstrated that string theory instanton corrections [14] modify the no-scale structure of GKP, leading to a supersymmetric $A d S_{4}$ vacuum with fixed $\rho$. Lastly, it was argued that, for suitable choice of parameters, anti-D3 brane would lift $A d S_{4}$ vacuum to a $d S_{4}$ background, with all moduli of the compactification manifold stabilized ${ }^{3}$. This is possible because $\overline{D 3}$ is a codimension zero defect in the effective four dimensional description, and thus violates the strong energy condition.

An obvious question of KKLT construction is whether effective description of $\overline{D 3}$ is indeed given by simple dimensional reduction of its ten dimensional effective action. The question is nontrivial, as KKLT model does not allow for a consistent ten dimensional lift: the Kähler modulus stabilization involves string instanton corrections, which are highly non-local from the ten dimensional perspective (assuming their ten dimensional description exists at all). Thus strictly speaking, there is no starting point for the derivation of the $\overline{D 3}$ effective action in KKLT model. To the best of our knowledge, there is no consistent derivation of the effective brane description in the Kaluza-Klein reduction for any compactification in the literature as well.

In section 2 we discuss a simple exactly soluble $D$-dimensional toy model in which we confirm that contrary to a supergravity mode, in a consistent KK reduction to $d<D-2$ dimensions, the energy condition of the extended positive tension $d$-dimensional defect indeed changes. The defect contribution to the $d$-dimensional vacuum energy $\Lambda_{d}$ is positive. The apparent conflict with the energy condition upon KK reduction is resolved because the defect introduction 'shifts' the stabilization value of the overall Kähler modulus of the compactification manifold. This is precisely what is observed in KKLT construction. However, though the vacuum energy in the toy model compactification increases, it can never become positive. The latter point is just a special case of the no-go theorem for supergravity with localized sources satisfying a BPS-like condition of Ref. [10]. Additionally, we explain the dangers of using incomplete set of $D$-dimensional equations of motion to deduce lower dimensional effective action.

In section 3 we discuss type IIB string theory flux compactifications. Though we work in the supergravity approximation with 3-branes and orientifold 3-planes only, we expect that our results can be extended to more general F-theory compactifications.

\footnotetext{
${ }^{3}$ Some general aspects of moduli fixing and potentials in string theory compactifications are also discussed in [15].
} 
We demonstrate that the same prescription as the one employed in section 2 leads to a consistent effective description of four dimensional gravity coupled to the overall Kähler modulus of the compactification manifold. 'Consistent' means that equations of motion derived from the effective action are equivalent to ten dimensional equations of motion. This resolves some puzzles of the consistent Kaluza-Klein compactifications of string theory with fluxes and localized sources raised in [7]. We conclude with discussion of partially integrating out supergravity modes from the lower dimensional effective action.

\section{Defect effective action in consistent $K K$ reductions}

In this section ${ }^{4}$ we consider a soluble toy model where we verify that in consistent Kaluza-Klein reductions the extended object lower dimensional effective action is obtained by integrating out the compact coordinates of the higher dimensional effective description.

Consider the following $D=d+q$ dimensional ${ }^{5}$ model of gravity coupled to the $q$-form field strength $F_{[q]}$

$$
S_{D}=\frac{1}{2 k_{D}^{2}} \int_{\mathcal{M}_{D}} d^{D} \xi \sqrt{-g}\left(R^{D}-\frac{1}{2 q !} F_{[q]}^{2}\right),
$$

where $k_{D}$ is $D$-dimensional gravitational coupling. We will use effective action (2.1) to study Kaluza-Klein reductions of direct warped product compactifications $\mathcal{M}_{D}=$ $\mathcal{M}_{d} \times S^{q}$ on $S^{q}$

$$
\begin{aligned}
d S_{D}^{2} & =g_{M N} d \xi^{M} d \xi^{N} \\
& =\sigma^{-2} \tilde{g}_{\mu \nu} d x^{\mu} d x^{\nu}+\sigma^{\frac{2(d-2)}{q}}\left(d S^{q}\right)^{2}
\end{aligned}
$$

where $\sigma=\sigma(x)$ is a $q$-sphere Kähler modulus, $\tilde{g}_{\mu \nu}=\tilde{g}_{\mu \nu}(x)$ is the metric on $\mathcal{M}_{d}$, and $\left(d S^{q}\right)^{2}$ is the metric on a round $S^{q}$. The metric ansatz (2.2) is supplemented with

$$
F_{[q]}=b \operatorname{vol}\left(S^{q}\right)
$$

Additionally, we will consider $N \gg 1, d$-dimensional neutral domain walls of tension $T_{d}>0$, uniformly distributed on $S^{q}$. Each domain wall has a DBI-type action

$$
S_{d}=-T_{d} \int_{\mathcal{M}_{d}} d^{d} x \sqrt{-\tilde{g}} \sigma^{-d}
$$

\footnotetext{
${ }^{4}$ Results reported in this section were obtained in collaboration with Rob Myers.

${ }^{5}$ We assume $d>2$.
} 
The total effective action is

$$
S_{t o t}=S_{D}+S_{(l o c)}=S_{D}+\sum_{i=1}^{N} S_{d},
$$

where the summation is over all localized defects on $S^{q}$.

Note that the stress tensor of the localized defects is

$$
T_{M N}^{(l o c)}=-\frac{2}{\sqrt{-g}} \frac{\delta S_{(l o c)}}{\delta g^{M N}},
$$

so

$$
T_{\mu \nu}^{(l o c)}=-\frac{T_{d} N}{\operatorname{vol}\left(S^{q}\right) \sigma^{d}} \tilde{g}_{\mu \nu}, \quad T_{m n}^{(l o c)}=0 .
$$

In $D$-dimensions, the stress tensor $T_{M N}^{l o c}$ satisfies the strong energy condition provided

$$
T_{t t}^{(l o c)}-\frac{1}{D-2} g_{t t} T_{K}^{K(l o c)}>0,
$$

which using (2.7) evaluates to

$$
-\frac{T_{d} N}{\operatorname{vol}\left(S^{q}\right) \sigma^{d}} \frac{q-2}{D-2} \tilde{g}_{t t}>0 .
$$

Clearly, codimension higher than two $(q>2)$ defects satisfy the strong energy condition, while defects of lower codimension violate it.

In what follows we assume that $\sigma$ is constant. We consider first $D$-dimensional solutions of (2.5), and show that they are equivalent to the solutions of the effective $d$-dimensional action, obtained by simply integrating out the $S^{q}$ coordinates in (2.5).

\section{$2.1 \quad D$-dimensional equations of motion}

With the flux ansatz (2.3), the $q$-form Bianchi identity are trivially satisfied. We are left with Einstein equations

$$
\begin{aligned}
R_{M N}= & \frac{1}{2(q-1) !}\left(F_{M \alpha_{2} \cdots \alpha_{q}} F_{N}{ }^{\alpha_{2} \cdots \alpha_{n}}-\frac{q-1}{q(d+q-2)} F_{[q]}^{2} g_{M N}\right) \\
& +k_{D}^{2}\left(T_{M N}^{l o c}-\frac{1}{d+q-2} T^{l o c} g_{M N}\right) .
\end{aligned}
$$

Eq. (2.10) for the components along the defect implies that $\mathcal{M}_{d}$ is an Einstein manifold

$$
r_{\mu \nu}^{(d)}=\Lambda_{d} \tilde{g}_{\mu \nu},
$$


with

$$
\Lambda_{d}=-\frac{b^{2}(q-1)}{2(d+q-2)} \sigma^{2(1-d)}-k_{D}^{2} \frac{T_{d} N}{\operatorname{vol}\left(S^{q}\right) \sigma^{d}} \frac{q-2}{d+q-2} .
$$

Note from (2.12) that as long as defects satisfy the strong energy condition in $D$ dimensions, i.e., $q>2$, in a consistent $\mathrm{KK}$ reductions they can not produce $d$-dimensional accelerating Universe. As we will show in section 3, this is a special case of a more general result that follows from a no-go theorem for supergravity compactifications with branes satisfying BPS-like condition of [10]. From (2.12) is appears that a positive tension defect satisfying a strong energy condition provides a negative contribution to $d$-dimensional cosmological constant. This conclusion would be correct, if before and after defect introduction the Kähler modulus of the compactification manifold (in this case $\sigma$ ) would not change. In fact, the Kähler modulus is shifted so that after all, the contribution of defect tension to $\Lambda_{d}$ is positive. Indeed, from the Einstein equations along the $S^{q}$ directions

$$
(q-1) \sigma^{2(2-d) / q}=\frac{b^{2}}{2} \frac{d-1}{(d+q-2)} \sigma^{2(2-d)}+k_{D}^{2} \frac{T_{d} N}{\operatorname{vol}\left(S^{q}\right) \sigma^{(d-2)}} \frac{d}{d+q-2} .
$$

From (2.13) it follows that defect introduction always results in the expansion of $S^{q}$, i.e., $\sigma$ increases. Using (2.12), (2.13),

$$
\Lambda_{d}=-\frac{b^{2}}{2 d} \frac{1}{\sigma^{2 d-2}}-\frac{(q-2)(q-1)}{d} \frac{1}{\sigma^{2(d+q-2) / q}}
$$

so, it is monotonically increasing function of $\sigma$ for $q>2$. Thus, introduction of localized sources (2.4) will always increase $\Lambda_{d}$.

\section{$2.2 d$-dimensional effective description}

We would like to reproduce the results of the previous section from the lower dimensional effective action, obtained by integrating out $S^{q}$ coordinates. For constant $\sigma$ and the metric and flux ansatze (2.2) and (2.3) correspondingly, we find

$$
\sqrt{-g}\left(R^{D}-\frac{1}{2 q !} F_{[q]}^{2}\right)=\sqrt{-\tilde{g}} \sqrt{g_{S^{q}}} \sigma^{-2}\left(r^{d} \sigma^{2}+(q-1) q \sigma^{2(2-d) / q}-\frac{1}{2} b^{2} \sigma^{4-2 d}\right) .
$$

Thus Kaluza-Klein reduction of (2.1) yields

$$
S_{D}^{e f f}=\frac{\operatorname{vol}\left(S^{q}\right)}{2 k_{D}^{2}} \int_{\mathcal{M}_{d}} d^{d} x \sqrt{-\tilde{g}}\left(r^{d}-\mathcal{V}_{b u l k}(\sigma)\right)
$$


where

$$
\mathcal{V}_{b u l k}=-(q-1) q \sigma^{2(2-d-q) / q}+\frac{1}{2} b^{2} \sigma^{2-2 d} .
$$

The KK reduction of the defect action $S_{(l o c)}$ is simply

$$
S_{(l o c)}^{e f f}=-N T_{d} \int_{\mathcal{M}_{d}} d^{d} x \sqrt{-\tilde{g}} \sigma^{-d} \equiv \int_{\mathcal{M}_{d}} d^{d} x \sqrt{-\tilde{g}}\left(-\mathcal{V}_{(l o c)}(\sigma)\right)
$$

Notice that the stress tensor computed from $S_{(l o c)}^{\text {eff }}$ violates the strong energy condition in $d$-dimensions. This is so because extended defects are now codimension zero objects. The complete effective action reads

$$
\begin{aligned}
S^{e f f} & =S_{D}^{e f f}+S_{(l o c)}^{e f f} \\
& =\frac{v o l\left(S^{q}\right)}{2 k_{D}^{2}} \int_{\mathcal{M}_{d}} d^{d} x \sqrt{-\tilde{g}}\left(r^{d}-\mathcal{V}(\sigma)\right)
\end{aligned}
$$

with

$$
\begin{aligned}
\mathcal{V} & =\mathcal{V}_{b u l k}+\mathcal{V}_{(l o c)} \\
& =-(q-1) q \sigma^{2(2-d-q) / q}+\frac{1}{2} b^{2} \sigma^{2-2 d}+k_{D}^{2} \frac{2 T_{d} N}{\operatorname{vol}\left(S^{q}\right) \sigma^{d}}
\end{aligned}
$$

It is easy to verify that effective action (2.19) equations of motion

$$
\begin{gathered}
r_{\mu \nu}^{d}-\frac{1}{2} r^{d} \tilde{g}_{\mu \nu}+\frac{1}{2} \mathcal{V} \tilde{g}_{\mu \nu}=0, \\
\frac{\partial \mathcal{V}}{\partial \sigma}=0,
\end{gathered}
$$

are equivalent to $D$-dimensional equations of motion (2.12), (2.13).

\subsection{Incorrect $d$-dimensional effective description}

In this section we illustrate the pitfalls of using incomplete $D$-dimensional equations of motion to deduce $d$-dimensional effective action. This approach was used in existing literature, and has led to quite unexpected conclusions concerning consistency of KK reductions in the type IIB flux compactifications.

For a constant modulus $\sigma$ we expect the effective action to be of the type (2.19), which we rewrite one more time as

$$
S^{e f f}=\frac{\operatorname{vol}\left(S^{q}\right)}{2 k_{D}^{2}} \int_{\mathcal{M}_{d}} d^{d} x \sqrt{-\tilde{g}}\left(r^{d}-\tilde{\mathcal{V}}(\sigma)\right) .
$$


Einstein equations from (2.23) imply that

$$
\begin{aligned}
& r_{\mu \nu}^{d}-\frac{1}{2} r^{d} \tilde{g}_{\mu \nu}+\frac{1}{2} \tilde{\mathcal{V}} \tilde{g}_{\mu \nu}=0, \\
& \frac{1}{2} \tilde{g}_{\mu \nu}\left(\tilde{\mathcal{V}}-(d-2) \Lambda_{d}\right)=0,
\end{aligned}
$$

where in the second line we used the fact that $\mathcal{M}_{d}$ must be an Einstein manifold. With (2.24), it is tempting to use $D$-dimensional equation of motion (2.12) to conclude

$$
\begin{aligned}
\tilde{\mathcal{V}} & =(d-2) \Lambda_{d} \\
& =-\frac{b^{2}(q-1)(d-2)}{2(d+q-2)} \sigma^{2(1-d)}-k_{D}^{2} \frac{T_{d} N}{\operatorname{vol}\left(S^{q}\right) \sigma^{d}} \frac{(d-2)(q-2)}{d+q-2} .
\end{aligned}
$$

Potential $\tilde{\mathcal{V}}$ (2.25) derived in this way disagrees with (2.20), and is manifestly incorrect. For one reason, in the absence of any defects $(N=0), \tilde{\mathcal{V}}$ implies that $\sigma$ can not be stabilized. This conclusion differs from exact $D$-dimensional analysis.

\section{KK reduction of type IIB supergravity with localized sources}

We now implement the computations of the toy model in the previous section in type IIB supergravity compactifications on a large six-dimensional manifold $\tilde{\mathcal{M}}_{6}$ with fluxes and localized 3-brane sources. Specifically, we obtain the correct potential for the overall Kähler modulus of $\tilde{\mathcal{M}}_{6}$. As the analysis are rather technical, we begin by stating the main conclusion. For the effective action obtained by integrating out the $\tilde{\mathcal{M}}_{6}$ coordinates from the ten dimensional type IIB supergravity action with branes, its equations of motion are equivalent to the ten dimensional equations of motion. To elucidate this agreement, one has to use the full set of type IIB equations of motion. This agreement is lost however is one evaluates the full effective action on the equations of motion for a subset of the supergravity modes, on-shell condition for which depends on the Kähler modulus. Such dependence is known to occur for the on-shell condition on the ten dimensional metric warp factors [10], and, as we argue below, also arises for the 3-form flux equations of motion.

As a byproduct of the analysis, we point out that for the nonvanishing four dimensional cosmological constant, the complex structure moduli of $\tilde{\mathcal{M}}_{6}$ can not be fixed at a point where the 3-form fluxes are imaginary-self-dual (ISD) [16]. The Kähler modulus potential derived here can be useful in study the backreaction of non-ISD fluxes. We hope to return to this problem in the future. 


\subsection{Conventions}

Consider a static ${ }^{6}$ type IIB string theory compactification on a large compact manifold $\mathcal{M}_{6}$ with fluxes. We assume the supergravity approximation is valid. In particular, we assume that all the relevant moduli are fixed at large values so that one can safely ignore $\alpha^{\prime}$ corrections.

The low-energy effective action is decomposed into two parts

$$
S_{\text {eff }}=S_{I I B}+S_{\text {sources }}
$$

where

$$
\begin{aligned}
S_{I I B}=\frac{1}{2 k_{10}^{2}} \int_{\mathcal{M}_{10}} & \left(R_{10} \wedge \star 1-\frac{1}{2} d \Phi \wedge \star d \Phi-e^{2 \Phi} d C_{(0)} \wedge \star d C_{(0)}-\frac{1}{2} e^{-\Phi} H_{3} \wedge \star H_{3}\right. \\
& \left.-\frac{1}{2} e^{\Phi} F_{3} \wedge \star F_{3}-\frac{1}{4} F_{5} \wedge \star F_{5}-\frac{1}{2} C_{(4)} \wedge H_{3} \wedge F_{3}\right)
\end{aligned}
$$

is the Einstein frame action of type IIB supergravity, and $S_{\text {sources }}$ is the effective action of the localized sources (branes wrapping various cycles of the compactification manifold). We take the ten dimensional space-time $\mathcal{M}_{10}$ to be a direct warped product of a four dimensional spacetime $\mathcal{M}_{4}$ and a six dimensional compact manifold $\tilde{\mathcal{M}}_{6}$. The Einstein frame metric is

$$
\begin{aligned}
d s_{10}^{2} & =\hat{g}_{M N} d \xi^{M} d \xi^{N} \\
& =e^{2 A(y)-6 u} g_{\mu \nu} d x^{\mu} d x^{\nu}+e^{-2 A(y)+2 u} \tilde{g}_{m n}(y) d y^{m} d y^{n},
\end{aligned}
$$

where $g_{\mu \nu}$ and $\tilde{g}_{m n}$ is a metric on $\mathcal{M}_{4}$ and $\tilde{\mathcal{M}}_{6}$ correspondingly, $A(y)$ is a warp factor, and $u$ defines the overall volume modulus of $\tilde{\mathcal{M}}_{6}$

$$
\sigma \equiv \operatorname{Im} \rho \equiv e^{4 u}
$$

As we review later, the choice of relative warpings by $e^{u}$ in (3.3) is required to decouple the fluctuations of $\rho(x)$ from the four dimensional metric fluctuations [17]. Additionally, there are nontrivial 5 -form $F_{5}$ and 3 -form

$$
G_{3}=F_{3}-\tau H_{3}
$$

fluxes. In (3.5) $\tau$ is the type IIB axiodilaton. $S_{\text {sources }}$ in (3.1) provides local sources (in addition to those in $\left.S_{I I B}\right)$ for the Einstein equations, Maxwell and dilaton equations.

\footnotetext{
${ }^{6}$ i.e., there are no "rolling" moduli.
} 
For example, for a $p$-brane wrapped on a $(p-3)$-cycle $\Sigma$ of the compactification manifold $\tilde{\mathcal{M}}_{6}$ we have

$$
S_{\text {sources }}=-T_{p} \int_{\mathcal{M}_{4} \times \Sigma} d^{p+1} \xi \sqrt{-g}+\mu_{p} \int_{\mathcal{M}_{4} \times \Sigma} C_{p+1},
$$

with

$$
T_{p}=\left|\mu_{p}\right| e^{(p-3) \Phi / 4} .
$$

We introduce the stress tensor $T_{M N}^{l o c}$ for the localized sources in (3.1)

$$
T_{M N}^{l o c}=-\frac{2}{\sqrt{-\hat{g}}} \frac{\delta\left(S_{\text {sources }}\right)}{\delta \hat{g}^{M N}} .
$$

In what follows we assume that the only localized sources are $D 3, \overline{D 3}$ branes, and orientifold 3-planes $O 3$.

We use a mostly positive convention for the signature $(-+\cdots+)$ and take $\epsilon_{1 \cdots 10}=$ +1 . The bosonic type IIB equations consist of the following [18]:

- The Einstein equations:

$$
R_{M N}=T_{M N}^{(1)}+T_{M N}^{(3)}+T_{M N}^{(5)}+k_{10}^{2}\left(T_{M N}^{l o c}-\frac{1}{8} \hat{g}_{M N} T^{l o c}\right),
$$

where the energy momentum tensors of the dilaton/axion field, $\mathcal{B}$, the three index antisymmetric tensor field, $\mathcal{F}_{(3)}$, and the self-dual five-index tensor field, $\mathcal{F}_{(5)}$, are given by

$$
\begin{gathered}
T_{M N}^{(1)}=P_{M} P_{N}{ }^{*}+P_{N} P_{M}{ }^{*}, \\
T_{M N}^{(3)}=\frac{1}{8}\left(G^{P Q}{ }_{M} G_{P Q N}^{*}+G^{* P Q}{ }_{M} G_{P Q N}-\frac{1}{6} g_{M N} G^{P Q R} G_{P Q R}^{*}\right),
\end{gathered}
$$

and

$$
T_{M N}^{(5)}=\frac{1}{6} \mathcal{F}^{P Q R S}{ }_{M} \mathcal{F}_{P Q R S N} .
$$

In the unitary gauge, $\mathcal{B}$ is a complex scalar field, and

$$
P_{M}=f^{2} \partial_{M} \mathcal{B}, \quad Q_{M}=f^{2} \operatorname{Im}\left(\mathcal{B} \partial_{M} \mathcal{B}^{*}\right),
$$

where

$$
f=\frac{1}{\left(1-\mathcal{B B}^{*}\right)^{1 / 2}},
$$

while the antisymmetric tensor field $G$ is given by

$$
G=f\left(\mathcal{F}_{(3)}-\mathcal{B F}_{(3)}^{*}\right)
$$


- The Maxwell equations:

$$
\left(\nabla^{P}-i Q^{P}\right) G_{M N P}=P^{P} G_{M N P}^{*}-\frac{2}{3} i \mathcal{F}_{M N P Q R} G^{P Q R} .
$$

- The dilaton equation:

$$
\left(\nabla^{M}-2 i Q^{M}\right) P_{M}=-\frac{1}{24} G^{P Q R} G_{P Q R} .
$$

- The self-dual equation:

$$
\mathcal{F}_{(5)}=\star \mathcal{F}_{(5)} .
$$

In addition, $\mathcal{F}_{(3)}$ and $\mathcal{F}_{(5)}$ satisfy Bianchi identities which follow from the definition of the field strengths in terms of their potentials:

$$
\begin{aligned}
& \mathcal{F}_{(3)}=d A_{(2)}, \\
& \mathcal{F}_{(5)}=d A_{(4)}-\frac{1}{8} \operatorname{Im}\left(A_{(2)} \wedge \mathcal{F}_{(3)}^{*}\right) .
\end{aligned}
$$

We would like to relate fluxes $\mathcal{F}_{(3)}, \mathcal{F}_{(5)}$, and the unitary dilaton $\mathcal{B}$ to the appropriate quantities of (3.2). This has been done, among many other places, in [19]:

$$
\begin{aligned}
\tau \equiv C_{(0)}+i e^{-\Phi} & =i \frac{1+\mathcal{B}}{1-\mathcal{B}}, \\
A_{(2)} & =C_{(2)}+i B_{(2)}, \\
A_{(4)} & =\frac{1}{4}\left(C_{(4)}+\frac{1}{2} B_{(2)} \wedge C_{(2)}\right),
\end{aligned}
$$

with

$$
F_{3}=d C_{(2)}, \quad H_{3}=d B_{(2)}
$$

\subsection{Ten dimensional equations of motion}

We begin with a more general metric ansatz (in Einstein frame)

$$
\begin{aligned}
d s_{10}^{2} & =\Omega_{1}^{2}(y) \omega_{1}^{2}(x) d s_{\mathcal{M}_{4}}^{2}(x)+\Omega_{2}^{2}(y) \omega_{2}^{2}(x) d s_{\tilde{\mathcal{M}}_{6}}^{2}(y) \\
& =\Omega_{1}^{2}(y) \omega_{1}^{2}(x) g_{\mu \nu}(x) d x^{\mu} d x^{\nu}+\Omega_{2}^{2}(y) \omega_{2}^{2}(x) \tilde{g}_{m n}(y) d y^{m} d y^{n}
\end{aligned}
$$

where $\mathcal{M}_{4}$ is taken to be an Einstein manifold, i.e.,

$$
r_{\mu \nu}^{(4)}(x)=\Lambda g_{\mu \nu}(x)
$$

and $\tilde{\mathcal{M}}_{6}$ is a six dimensional compactification manifold. Additionally we assume that all the stringy matter and the localized sources depend on $y$ only. The rigorous way to 
say this, is that we require our compactification to preserve (anti-)de-Sitter or Poincare invariance. For the 5 -form $\mathcal{F}_{5}$ we assume

$$
\mathcal{F}_{5}=(1+\star)\left[d \omega \wedge \operatorname{vol}_{\mathcal{M}_{4}}\right]
$$

where $\operatorname{vol}_{\mathcal{M}_{4}}$ is the volume form on $\mathcal{M}_{4}$. The basic idea is to allow $\Lambda$ in (3.23) to "dynamically adjust" (letting it be either positive, zero or negative) depending on the fluxes and the geometry of the compact $\tilde{\mathcal{M}}_{6}$. We should say that the equations we obtain are equivalent (when they can be compared) to those presented in [10]. To elucidate the agreement with [10], some redefinitions are necessary. For example, the $G_{M N P}$ we are using is related to the $G_{3}$ flux (3.5) of [10] as

$$
G_{3}=\frac{1}{f(1-\mathcal{B})} G
$$

In what follows all the index contractions are done with the unwarped metrics $g_{\mu \nu}, \tilde{g}_{m n}$. Also we use $r_{\mu \nu}^{(4)}$ to be the Ricci tensor of $\mathcal{M}_{4}$, and $r_{m n}^{(6)}$ for the Ricci tensor of $\tilde{\mathcal{M}}_{6}$. Capital $R_{\mu \nu}, R_{m n}$ are reserved for the Ricci components of the full ten dimensional warped metric (3.22). The absence of indexes implies that they are contracted. After straightforward computations we find

$$
\begin{aligned}
R_{\mu \nu}= & r_{\mu \nu}^{(4)}+2\left(2 \omega_{1}^{-2} \nabla_{\mu} \omega_{1} \nabla_{\nu} \omega_{1}-\omega_{1}^{-1} \nabla_{\mu} \nabla_{\nu} \omega_{1}\right) \\
& +6\left(\omega_{1}^{-1} \omega_{2}^{-1}\left[\nabla_{\mu} \omega_{1} \nabla_{\nu} \omega_{2}+\nabla_{\mu} \omega_{2} \nabla_{\nu} \omega_{1}\right]-\omega_{2}^{-1} \nabla_{\mu} \nabla_{\nu} \omega_{2}\right) \\
& -g_{\mu \nu}\left(\omega_{1}^{-1} \nabla^{2} \omega_{1}+\omega_{1}^{-2}\left(\nabla \omega_{1}\right)^{2}+6 \omega_{1}^{-1} \omega_{2}^{-1} \nabla \omega_{1} \nabla \omega_{2}+\Omega_{2}^{-2} \omega_{2}^{-2} \Omega_{1} \omega_{1}^{2} \tilde{\nabla}^{2} \Omega_{1}\right. \\
& \left.+3 \Omega_{2}^{-2} \omega_{2}^{-2} \omega_{1}^{2}\left(\tilde{\nabla} \Omega_{1}\right)^{2}+4 \Omega_{2}^{-3} \omega_{2}^{-2} \Omega_{1} \omega_{1}^{2} \tilde{\nabla} \Omega_{1} \tilde{\nabla} \Omega_{2}\right)
\end{aligned}
$$

where

$$
\nabla \equiv \nabla_{x}, \quad \tilde{\nabla} \equiv \nabla_{y}
$$

and

$$
\begin{aligned}
R_{m n}= & r_{m n}^{(6)}+4\left(2 \Omega_{2}^{-2} \tilde{\nabla}_{m} \Omega_{2} \tilde{\nabla}_{n} \Omega_{2}-\Omega_{2}^{-1} \tilde{\nabla}_{m} \tilde{\nabla}_{n} \Omega_{2}\right) \\
& +4\left(\Omega_{1}^{-1} \Omega_{2}^{-1}\left[\tilde{\nabla}_{m} \Omega_{1} \tilde{\nabla}_{n} \Omega_{2}+\tilde{\nabla}_{m} \Omega_{2} \tilde{\nabla}_{n} \Omega_{1}\right]-\Omega_{1}^{-1} \tilde{\nabla}_{m} \tilde{\nabla}_{n} \Omega_{1}\right) \\
& -\tilde{g}_{m n}\left(\Omega_{2}^{-1} \tilde{\nabla}^{2} \Omega_{2}+3 \Omega_{2}^{-2}\left(\tilde{\nabla} \Omega_{2}\right)^{2}+4 \Omega_{1}^{-1} \Omega_{2}^{-1} \tilde{\nabla} \Omega_{1} \tilde{\nabla} \Omega_{2}\right. \\
& \left.+\Omega_{1}^{-2} \omega_{1}^{-2} \Omega_{2}^{2} \omega_{2} \nabla^{2} \omega_{2}+5 \Omega_{1}^{-2} \omega_{1}^{-2} \Omega_{2}^{2}\left(\nabla \omega_{2}\right)^{2}+2 \omega_{1}^{-3} \omega_{2} \Omega_{1}^{-2} \Omega_{2}^{2} \nabla \omega_{1} \nabla \omega_{2}\right)
\end{aligned}
$$


Also, $R_{\mu m} \neq 0$, but we will not need its explicit form in what follows. The choice of warp factors as in (3.3),

$$
\begin{aligned}
& \Omega_{1}=e^{A(y)}, \quad \Omega_{2}=e^{-A(y)}, \\
& \omega_{1}=e^{-3 u(x)}, \quad \omega_{2}=e^{u(x)},
\end{aligned}
$$

substantially simplifies computations. With (3.26)-(3.29) we find

$$
\begin{aligned}
R_{10} \sqrt{-\hat{g}}= & e^{-4 A} \sqrt{\tilde{g}} \sqrt{-g}\left(r^{(4)}+6 \nabla^{2} u-24(\nabla u)^{2}\right) \\
& +e^{-8 u} \sqrt{\tilde{g}} \sqrt{-g}\left(r^{(6)}+2 \tilde{\nabla}^{2} A-8(\tilde{\nabla} A)^{2}\right) .
\end{aligned}
$$

Note that terms containing $\nabla^{2} u$ and $\tilde{\nabla}^{2} A$ are total derivatives and can be dropped from the effective action. From (3.30), the four dimensional gravitational constant $k_{4}$ in terms of ten dimensional gravitational constant $k_{10}$ is given by

$$
\frac{1}{k_{4}^{2}}=\frac{1}{k_{10}^{2}} \int_{\tilde{\mathcal{M}}_{6}} \sqrt{\tilde{g}} e^{-4 A} .
$$

Thus, as in $[10,17]$, we get from $(3.30)$ the kinetic term for the $\operatorname{Im} \rho \equiv e^{4 u}$ modulus

$$
\frac{1}{k_{4}^{2}} \int_{\mathcal{M}_{4}} d^{4} x \sqrt{-g}\left\{-3 \frac{\nabla \bar{\rho} \nabla \rho}{|\rho-\bar{\rho}|^{2}}\right\},
$$

corresponding to the Kähler potential

$$
\mathcal{K}_{\rho}=-3 \ln (-i(\rho-\bar{\rho}))
$$

From now on we assume that the modulus $\rho$ is constant over $\mathcal{M}_{4}$. Note that with $\rho$ being constant

$$
R_{\mu m}=0
$$

From (3.6), (3.8) localized stress tensor contribution from 3 -branes, $T_{M N}^{3-\text { brane }}$, is

$$
T_{\mu \nu}^{3-\text { brane }}=-T_{3} e^{2 A}(\operatorname{Im} \rho)^{-3 / 2} g_{\mu \nu} \rho_{3}^{\text {loc }}, \quad T_{m n}^{3-\text { brane }}=0,
$$

where $\rho_{3}$ is the number density of 3-branes:

$$
\#(3-\text { branes })=\int_{\mathcal{M}_{6}} d^{6} y \sqrt{\tilde{g}} e^{-6 A}(\operatorname{Im} \rho)^{3 / 2} \rho_{3}^{l o c} .
$$

For a single 3 -brane localized at $y=y_{0}$ we have

$$
\rho_{3}^{l o c}=e^{6 A}(\operatorname{Im} \rho)^{-3 / 2} \frac{\delta^{6}\left(y-y_{0}\right)}{\sqrt{\tilde{g}}} .
$$


Since $O 3$ tension is $-\frac{1}{4} T_{3}$, contribution to the localized stress tensor from a single $O 3$ plane will be minus a quarter of that of a $D 3$ brane. To treat orientifold planes and 3-branes on the same footing we write the full localized stress tensor as

$$
T_{\mu \nu}^{l o c}=-e^{8 A}(\operatorname{Im} \rho)^{-3} g_{\mu \nu} \sum_{\text {sources }} T_{i} \frac{\delta^{6}\left(y-y_{i}\right)}{\sqrt{\tilde{g}}}, \quad T_{m n}^{l o c}=0,
$$

where the summation is over all sources localized at $y=\left\{y_{i}\right\}$ and having tension

$$
\begin{aligned}
T_{i} \equiv T_{3}, & \text { source }_{i} & \equiv\{D 3, \overline{D 3}\}, \\
T_{i} \equiv-\frac{1}{4} T_{3}, & \text { source }_{i} & \equiv\{O 3\} .
\end{aligned}
$$

The simplest Einstein equation is for the $\{\mu \nu\}$ components

$$
\begin{aligned}
\Lambda-(\operatorname{Im} \rho)^{-2} e^{4 A} \tilde{\nabla}^{2} A= & -(\operatorname{Im} \rho)^{-3} \frac{1}{48} G_{m n p} \bar{G}^{m n p} e^{8 A}-(\operatorname{Im} \rho)^{4} 4(\tilde{\nabla} \omega)^{2} e^{-4 A} \\
& -\frac{1}{2} k_{10}^{2} e^{8 A}(\operatorname{Im} \rho)^{-3} \sum_{\text {sources }} T_{i} \frac{\delta^{6}\left(y-y_{i}\right)}{\sqrt{\tilde{g}}},
\end{aligned}
$$

or

$$
\begin{aligned}
\tilde{\nabla}^{2} e^{4 A}= & (\operatorname{Im} \rho)^{-1} \frac{1}{12} e^{8 A} G \bar{G}+e^{-4 A}\left(16(\operatorname{Im} \rho)^{6}(\tilde{\nabla} \omega)^{2}+\left(\tilde{\nabla} e^{4 A}\right)^{2}\right) \\
& +(\operatorname{Im} \rho)^{2}\left(4 \Lambda+2 k_{10}^{2} e^{8 A}(\operatorname{Im} \rho)^{-3} \sum_{\text {sources }} T_{i} \frac{\delta^{6}\left(y-y_{i}\right)}{\sqrt{\tilde{g}}}\right)
\end{aligned}
$$

Note the shorthand notation for $G \bar{G}$.

The next simplest equation is for the 5 -form Bianchi identity

$$
\begin{aligned}
(\operatorname{Im} \rho)^{3} \tilde{\nabla}^{2} \omega= & (\operatorname{Im} \rho)^{3} 2 e^{-4 A} \tilde{\nabla} \omega \tilde{\nabla} e^{4 A}+(\operatorname{Im} \rho)^{-1} \frac{i}{48} e^{8 A} G \star_{6} \bar{G} \\
& -\frac{1}{2} k_{10}^{2} e^{8 A}(\operatorname{Im} \rho)^{-1} \sum_{\text {sources }} q_{i}\left|T_{i}\right| \frac{\delta^{6}\left(y-y_{i}\right)}{\sqrt{\tilde{g}}},
\end{aligned}
$$

where $\star_{6}$ is defined on manifold $\tilde{\mathcal{M}}_{6}$, and $q_{i}= \pm 1$ is a charge of a localized source at $y=y_{i}$. Note that above normalization of $q_{i}$ is correct for $O 3$ planes as well: their 'physical' charge is $\left(-\frac{1}{4}\right)$ of a $D 3$ brane charge.

From (3.41) and (3.42) we find an important constraint - a simple modification of eq. $(2.30)$ of $[10]$

$$
\begin{gathered}
\tilde{\nabla}^{2}\left((\operatorname{Im} \rho)^{3} 4 \omega+e^{4 A}\right)=e^{-4 A}\left(\tilde{\nabla}\left[(\operatorname{Im} \rho)^{3} 4 \omega+e^{4 A}\right]\right)^{2}+(\operatorname{Im} \rho)^{-1} \frac{1}{24} e^{8 A}\left|i G+\star_{6} G\right|^{2} \\
+(\operatorname{Im} \rho)^{2} 4 \Lambda+(\operatorname{Im} \rho)^{-1} 2 k_{10}^{2} e^{8 A} \sum_{\text {sources }}\left(T_{i}-q_{i}\left|T_{i}\right|\right) \frac{\delta^{6}\left(y-y_{i}\right)}{\sqrt{\tilde{g}}}
\end{gathered}
$$


To proceed with the remaining equations of motion we separate imaginary (anti-) self dual parts of $G$ :

$$
\begin{aligned}
G^{+} & \equiv \frac{1}{2} G-\frac{i}{2} \star_{6} G, \\
G^{-} & \equiv \frac{1}{2} G+\frac{i}{2} \star_{6} G,
\end{aligned}
$$

where

$$
\star_{6} G^{ \pm}= \pm i G^{ \pm} .
$$

It will also be convenient to introduce a 3 -form $\mathcal{L}$

$$
\mathcal{L}=e^{4 A} \star_{6} G-4 i \omega(\operatorname{Im} \rho)^{3} G=-i\left(e^{4 A}+4 \omega(\operatorname{Im} \rho)^{3}\right) G^{-}+i\left(e^{4 A}-4 \omega(\operatorname{Im} \rho)^{3}\right) G^{+} .
$$

The remaining Einstein equations are

$$
\begin{aligned}
r_{m n}^{(6)}= & \frac{1}{2} e^{-8 A}\left(\tilde{\nabla}_{m} e^{4 A} \tilde{\nabla}_{n} e^{4 A}-16(\operatorname{Im} \rho)^{6} \tilde{\nabla}_{m} \omega \tilde{\nabla}_{n} \omega\right)-\Lambda(\operatorname{Im} \rho)^{2} e^{-4 A} \tilde{g}_{m n}+T_{m n}^{(1)} \\
& +(\operatorname{Im} \rho)^{-1} \frac{1}{4} e^{4 A}\left(G^{+}{ }_{p q m} \bar{G}^{-p q}{ }_{n}+G^{-}{ }_{p q m} \bar{G}^{+p q}{ }_{n}\right),
\end{aligned}
$$

where $T_{m n}^{(1)}$ is the standard dilaton/axion stress tensor

$$
T_{m n}^{(1)}=\frac{1}{4} \frac{\tilde{\nabla}_{m} \tau \tilde{\nabla}_{n} \bar{\tau}+\tilde{\nabla}_{n} \tau \tilde{\nabla}_{m} \bar{\tau}}{(\operatorname{Im} \tau)^{2}}
$$

The 3-form Maxwell equations (3.16) are reduced to

$$
0=d \mathcal{L}+f^{2}\left(\overline{\mathcal{L}} \wedge d \mathcal{B}+\frac{1}{2} \mathcal{L} \wedge(\mathcal{B} d \overline{\mathcal{B}}-\overline{\mathcal{B}} d \mathcal{B})\right) .
$$

Finally, the dilaton equation is

$$
f^{2} \tilde{\nabla}^{2} \mathcal{B}+2 f^{4} \overline{\mathcal{B}}(\tilde{\nabla} \mathcal{B})^{2}=-\frac{1}{12} e^{6 A} G^{+} G^{-} .
$$

Integrating both sides of (3.43) over compact manifold $\tilde{\mathcal{M}}_{6}$ we find

$$
\begin{aligned}
\Lambda & =\left\{-\frac{1}{4(\operatorname{Im} \rho)^{2} \operatorname{vol}_{\tilde{\mathcal{M}}_{6}}} \int_{\tilde{\mathcal{M}}_{6}} d^{6} y \sqrt{\tilde{g}}\left(e^{-4 A}\left(\tilde{\nabla}\left[(\operatorname{Im} \rho)^{3} 4 \omega+e^{4 A}\right]\right)^{2}\right.\right. \\
& \left.\left.+(\operatorname{Im} \rho)^{-1} \frac{1}{6} e^{8 A}\left|G^{+}\right|^{2}\right)\right\} \\
& +\left\{-\frac{k_{10}^{2}}{2(\operatorname{Im} \rho)^{3} \text { vol }_{\tilde{\mathcal{M}}_{6}}} \sum_{\text {sources }} e^{8 A\left(y_{i}\right)}\left(T_{i}-q_{i}\left|T_{i}\right|\right)\right\},
\end{aligned}
$$


where we separated contributions to the four dimensional cosmological constant coming from supergravity modes, and the localized sources. Also,

$$
\operatorname{vol}_{\tilde{\mathcal{M}}_{6}}=\int_{\tilde{\mathcal{M}}_{6}} d^{6} y \sqrt{\tilde{g}}
$$

We would like to conclude the section with several comments concerning solutions of equations of motion derived here.

- First of all, notice that the only contribution to the four dimensional cosmological constant from localized sources comes from $\overline{D 3}$ branes. Both D3 branes and $O 3$ planes saturate the BPS-like bound of GKP (which can be thought of as a generalized strong energy condition for charged defects in ten dimensions), and do not contribute to $\Lambda . \overline{D 3}$ branes satisfy this bound, and thus provide negative contribution to $\Lambda$. As the result, supergravity plus localized 3-branes and orientifold 3-planes flux compactifications can never lead to four dimensional de Sitter. This result is straightforward to generalize to all extended objects in string theory satisfying the BPS-like condition of GKP. Note that the neutral defects in the toy model of section 2 satisfy the GKP bound.

To circumvent this no-go theorem for de-Sitter compactifications, one possibility is to consider flux compactifications with localized sources that violate BPS-like condition of GKP. These include $\overline{O 3}$ planes and orientifold 5-planes, O5. Currently no construction of this type is known. Another possibility is to relax the condition that all moduli of the compactification manifold are stabilized. There are interesting cosmological solutions with rolling moduli [6]. However, to our knowledge, no nonsingular solution of this type (in supergravity with branes) exists. Most promising appear to be the framework of KKLT [8], where one first employs nonperturbative string corrections to stabilize all moduli with supersymmetric $A d S_{4}$ vacuum, and further lifts it to a $d S_{4}$ with supersymmetry breaking branes. Here, it is important to better understand effects of the supersymmetry breaking, in particular the role of non-ISD fluxes that can be induced by these supersymmetry breaking effects.

- The 3-form Maxwell equation (3.49) allow for a solution

$$
\mathcal{L}=0
$$

We do not know whether (3.53) gives the most general solution of the 3-form Maxwell equation (3.49), though we suspect this is the case. The string theory flux backgrounds constraint by (3.53) include those discussed in [10,3]. This is the class of compactifications we concentrate on here. Notice that (3.53) does not imply that the 3-form flux 
is ISD, i.e.,

$$
G^{+}=0 \text {. }
$$

In fact, this is simply inconsistent once $\Lambda \neq 0$. Indeed, given (3.54), eqs. (3.46), (3.53) then imply that $e^{4 A}+4 \omega(\operatorname{Im} \rho)^{3}=0$. This last condition, along with $G^{+}=0$, implies from (3.43)

$$
0=(\operatorname{Im} \rho)^{2} 4 \Lambda+(\operatorname{Im} \rho)^{-1} 2 k_{10}^{2} e^{8 A} \sum_{\text {sources }}\left(T_{i}-q_{i}\left|T_{i}\right|\right) \frac{\delta^{6}\left(y-y_{i}\right)}{\sqrt{\tilde{g}}} .
$$

Clearly, (3.55) can not be satisfied locally, given that cosmological constant $\Lambda$ is uniform over $\tilde{\mathcal{M}}_{6}$.

- It is tempting to use (3.51), and follow the arguments of Sec. 2.3, to conclude that the Kähler modulus potential $\tilde{\mathcal{V}}(\rho)$ is

$$
\begin{aligned}
\tilde{\mathcal{V}}(\rho)= & 2 \Lambda \\
= & \left\{-\frac{1}{2(\operatorname{Im} \rho)^{2} \operatorname{vol}_{\tilde{\mathcal{M}}_{6}}} \int_{\tilde{\mathcal{M}}_{6}} d^{6} y \sqrt{\tilde{g}}\left(e^{-4 A}\left(\tilde{\nabla}\left[(\operatorname{Im} \rho)^{3} 4 \omega+e^{4 A}\right]\right)^{2}\right.\right. \\
& \left.\left.+(\operatorname{Im} \rho)^{-1} \frac{1}{6} e^{8 A}\left|G^{+}\right|^{2}\right)\right\} \\
& +\left\{-\frac{k_{10}^{2}}{(\operatorname{Im} \rho)^{3} \operatorname{vol}_{\tilde{\mathcal{M}}_{6}}} \sum_{\text {sources }} e^{8 A\left(y_{i}\right)}\left(T_{i}-q_{i}\left|T_{i}\right|\right)\right\} .
\end{aligned}
$$

This is incorrect, as the use of $\tilde{\mathcal{V}}$ in the effective action analogous to (2.23) implies that $\rho$ can be off-shell; because of this, this Kähler potential can never be deduces from on-shell quantities, like equations of motion. The exactly soluble toy model of section 2 explicitly demonstrates this point.

- We would like to present a simple expression for $\Lambda$, following from the full set of ten dimensional equations of motion

$$
\Lambda=-\frac{k_{4}^{2}}{2} \sum_{\text {sources }}\left(T_{i} e^{4 A\left(y_{i}\right)}(\operatorname{Im} \rho)^{-3}+4 q_{i}\left|T_{i}\right| \omega\left(y_{i}\right)\right) .
$$

The derivation of (3.57) is delegated to Appendix. It would be nice to find a simpler derivation of (3.57); in particular it's extension for more general F-theory flux compactifications.

Notice that (3.57) suggests that the four dimensional cosmological constant is sensitive to exactly how $\overline{D 3}$ branes are distributed ${ }^{7}$ between different Klebanov-Strassler-like

\footnotetext{
${ }^{7}$ As explained in KKLT, $\overline{D 3}$ branes are driven to the end of the 'Klebanov-Strassler throat' of $\tilde{\mathcal{M}}_{6}$,
} where the warp factor $e^{A}$ is locally minimized. 
'throats' of $\tilde{\mathcal{M}}_{6}$. This opens a possibility of interesting cosmological transitions where in evolution to global minimum $\Lambda$, the Universe experiences metastable vacua with different cosmological constants ${ }^{8}$. We plan to study these transitions in the future.

\subsection{Four dimensional effective description}

In this section we derive the four dimensional effective description of supergravity and localized sources flux compactifications, implementing the arguments of Sec. 2.2. We find that equations of motion derived from this effective description are equivalent to a subset of ten dimensional equations of motion. The Kähler modulus potential is given by Eq. (3.65).

Recall that (3.2)

$$
\begin{aligned}
S_{I I B}= & \frac{1}{2 k_{10}^{2}} \int_{\mathcal{M}_{10}} d^{10} \xi \sqrt{-\hat{g}}\left\{R_{10}-\frac{\partial_{M} \tau \partial^{M} \bar{\tau}}{2(\operatorname{Im} \tau)^{2}}-\frac{G \cdot \bar{C}}{12}-\frac{F_{(5)}^{2}}{4 \cdot 5 !}\right\} \\
& +\frac{1}{8 i k_{10}^{2}} \int_{\mathcal{M}_{10}} C_{(4)} \wedge G \wedge \bar{G} .
\end{aligned}
$$

Let's evaluate the ten dimensional effective action (3.1) for the metric ansatz (3.22), (3.29). We rewrite (3.1) as

$$
S_{e f f}^{(4)}=S_{I I B}^{(4)}+S_{\text {sources }}^{(4)}
$$

where

$$
\begin{aligned}
S_{I I B}^{(4)}= & \frac{1}{2 k_{10}^{2}} \int_{\mathcal{M}_{4}} d^{4} x \sqrt{-g} \int_{\tilde{\mathcal{M}}_{6}} d^{6} y \sqrt{\tilde{g}}\left(r^{(4)} e^{-4 A(y)}\right. \\
& +\frac{1}{(\operatorname{Im} \rho)^{2}}\left[r^{(6)}(y)-8(\tilde{\nabla} A(y))^{2}-\frac{(\tilde{\nabla} \tau(y))^{2}}{2(\operatorname{Im} \tau)^{2}}\right]-\frac{1}{12(\operatorname{Im} \rho)^{3}} e^{4 A(y)} G \cdot \bar{G}(y) \\
& \left.+8 e^{-8 A(y)}(\operatorname{Im} \rho)^{4}(\tilde{\nabla} \omega(y))^{2}\right)+C S,
\end{aligned}
$$

where we used (3.20) and $(3.24)^{9}$. CS denotes the type IIB supergravity Chern-Simons

\footnotetext{
${ }^{8}$ Related issues have been discussed in [20].

${ }^{9}$ One has to be careful with evaluation of the action of the self-dual 5-form. A correct prescription to do this was explained in [17].
} 
term, see (3.58). Explicitly,

$$
\begin{aligned}
C S & =\frac{1}{8 i k_{10}^{2}} \int_{\mathcal{M}_{10}} 8 \omega \operatorname{vol}_{\mathcal{M}_{4}} \wedge G \wedge \bar{G} \\
& =\frac{1}{2 k_{10}^{2}} \int_{\mathcal{M}_{4}} d^{4} x \sqrt{-g} \int_{\tilde{\mathcal{M}}_{6}} d^{6} y \sqrt{\tilde{g}}\left(\frac{i \omega}{3} G \cdot \star_{6} \bar{G}\right) .
\end{aligned}
$$

Note an extra factor of 2 for the $C_{(4)}$ in (3.61) — again, this is required for proper dimensional reduction of the self-dual 5-form, [17]. Thus (3.60) reads

$$
\begin{aligned}
S_{I I B}^{(4)}= & \frac{1}{2 k_{10}^{2}} \int_{\mathcal{M}_{4}} d^{4} x \sqrt{-g} \int_{\tilde{\mathcal{M}}_{6}} d^{6} y \sqrt{\tilde{g}}\left(r^{(4)} e^{-4 A(y)}\right. \\
& +\frac{1}{(\operatorname{Im} \rho)^{2}}\left[r^{(6)}(y)-8(\tilde{\nabla} A(y))^{2}-\frac{(\tilde{\nabla} \tau(y))^{2}}{2(\operatorname{Im} \tau)^{2}}\right]+8 e^{-8 A(y)}(\operatorname{Im} \rho)^{4}(\tilde{\nabla} \omega(y))^{2} \\
& \left.+\frac{1}{12(\operatorname{Im} \rho)^{3}} G \cdot \star_{6} \overline{\mathcal{L}}(y)\right) .
\end{aligned}
$$

Finally, the dimensional reduction of the localized sources (again, only $D 3, \overline{D 3}$ branes and $O 3$ planes) (3.6) reads

$$
S_{\text {sources }}^{(4)}=-\sum_{\text {sources }}\left(T_{i} e^{4 A\left(y_{i}\right)}(\operatorname{Im} \rho)^{-3}+4 q_{i}\left|T_{i}\right| \omega\left(y_{i}\right)\right) \int_{\mathcal{M}_{4}} d^{4} x \sqrt{-g} .
$$

Now, rewriting effective action (3.59) as

$$
S_{\text {eff }}^{(4)}=\frac{1}{2 k_{4}^{2}} \int_{\mathcal{M}_{4}} d^{4} x \sqrt{-g}\left(r^{(4)}-\mathcal{V}(\rho)\right),
$$

we conclude from (3.62) and (3.63)

$$
\begin{aligned}
\mathcal{V}(\rho)= & -(\operatorname{Im} \rho)^{-2} \frac{k_{4}^{2}}{k_{10}^{2}} \int_{\tilde{\mathcal{M}}_{6}} d^{6} y \sqrt{\tilde{g}}\left[r^{(6)}(y)-8(\tilde{\nabla} A(y))^{2}-\frac{(\tilde{\nabla} \tau(y))^{2}}{2(\operatorname{Im} \tau)^{2}}\right] \\
& -(\operatorname{Im} \rho)^{4} \frac{k_{4}^{2}}{k_{10}^{2}} \int_{\tilde{\mathcal{M}}_{6}} d^{6} y \sqrt{\tilde{g}}\left[8 e^{-8 A(y)}(\tilde{\nabla} \omega(y))^{2}\right] \\
& +(\operatorname{Im} \rho)^{-3} \frac{k_{4}^{2}}{k_{10}^{2}} \int_{\tilde{\mathcal{M}}_{6}} d^{6} y \sqrt{\tilde{g}}\left[\frac{1}{12} e^{4 A(y)} G \cdot \bar{G}(y)\right] \\
& -\frac{k_{4}^{2}}{k_{10}^{2}} \int_{\tilde{\mathcal{M}}_{6}} d^{6} y \sqrt{\tilde{g}}\left[\frac{i \omega}{3} G \cdot \star_{6} \bar{G}\right] \\
& +2 k_{4}^{2} \sum_{\text {sources }}\left(T_{i} e^{4 A\left(y_{i}\right)}(\operatorname{Im} \rho)^{-3}+4 q_{i}\left|T_{i}\right| \omega\left(y_{i}\right)\right)
\end{aligned}
$$


where we organized contributions according to various scaling with $(\operatorname{Im} \rho) \equiv \sigma$ :

$$
\mathcal{V}(\rho) \equiv \mathcal{V}(\sigma) \equiv \sigma^{-2} V_{-2}+\sigma^{4} V_{4}+\sigma^{-3} V_{-3}+V_{0}+V_{\text {sources }},
$$

where $\left\{V_{-2}, V_{4}, V_{-3}, V_{0}, V_{\text {soruces }}\right\}$ can be read off comparing (3.65) and (3.66). We would like to claim that (3.65) is the correct off-shell potential for $\rho$.

Consider Einstein equations derived from (3.64). They imply that $\mathcal{M}_{4}$ is an Einstein manifold

$$
r_{\mu \nu}^{(4)}=\Lambda^{(4)} g_{\mu \nu}
$$

with

$$
\Lambda^{(4)}=\frac{1}{2} \mathcal{V}(\sigma)
$$

We demonstrate now that $\Lambda^{(4)}$ is actually equivalent to ten dimensional expression (3.57), once ten dimensional on-shell condition, i.e., equations of motion, is used. Indeed, because of (3.53),

$$
\sigma^{-3} V_{-3}+V_{0}=0
$$

Now the trace of (3.47) implies

$$
(\operatorname{Im} \rho)^{-2}\left[r^{(6)}-8(\tilde{\nabla} A)^{2}-\frac{(\tilde{\nabla} \tau)^{2}}{2(\operatorname{Im} \tau)^{2}}\right]+(\operatorname{Im} \rho)^{4}\left[8 e^{-8 A}(\tilde{\nabla} \omega)^{2}\right]=-6 \Lambda e^{-4 A}
$$

or, equivalently,

$$
\sigma^{-2} V_{-2}+\sigma^{4} V_{4}=6 \Lambda
$$

Using (3.57), we conclude that

$$
V_{\text {sources }}=-4 \Lambda \text {. }
$$

Given (3.69), (3.71), (3.72) we conclude from (3.66)

$$
\mathcal{V}(\sigma)=2 \Lambda
$$

which along with (3.68) gives

$$
\Lambda^{(4)}=\Lambda
$$

In the rest of this section we show that the equation of motion for the Kähler modulus $\sigma$

$$
\frac{d \mathcal{V}(\sigma)}{d \sigma} \equiv \mathcal{V}^{\prime}=0
$$


is satisfied as well. From (3.66),

$$
\sigma \mathcal{V}^{\prime}=-2 \sigma^{-2} V_{-2}+4 \sigma^{4} V_{4}-3 \sigma^{-3} V_{-3}-6 k_{4}^{2} \sum_{\text {sources }} T_{i} e^{4 A\left(y_{i}\right)} \sigma^{-3},
$$

where the last term comes from the derivative of $V_{\text {sources }}$. Using (3.71), Eq. (3.76) becomes

$$
\sigma \mathcal{V}^{\prime}=-12 \Lambda+6 \sigma^{4} V_{4}-3 \sigma^{-3} V_{-3}-6 k_{4}^{2} \sum_{\text {sources }} T_{i} e^{4 A\left(y_{i}\right)} \sigma^{-3} .
$$

Notice from (3.85)

$$
\Lambda=-\frac{k_{4}^{2}}{2} \sum_{\text {sources }} T_{i} e^{4 A\left(y_{i}\right)} \sigma^{-3}-\frac{1}{4} \sigma^{-3} V_{-3}+\frac{1}{2} \sigma^{4} V_{4},
$$

thus Eq. (3.77) becomes

$$
\begin{aligned}
\sigma \mathcal{V}^{\prime} & =-12 \Lambda+\left(12 \Lambda+6 k_{4}^{2} \sum_{\text {sources }} T_{i} e^{4 A\left(y_{i}\right)} \sigma^{-3}\right)-6 k_{4}^{2} \sum_{\text {sources }} T_{i} e^{4 A\left(y_{i}\right)} \sigma^{-3} \\
& =0
\end{aligned}
$$

\subsection{Concluding remarks}

We would like to emphasize that to obtain agreement between ten dimensional and four dimensional effective descriptions of string theory flux compactifications ${ }^{10}$ one has to use the full potential (3.65). In particular, it is incorrect to evaluate compact supergravity modes on their equations of motion in the expression for $\mathcal{V}(\sigma)$. This was explicitly demonstrated in Sec. 2.3 for the case of the exactly soluble toy model.

Similar phenomenon can be observed in the current context. Indeed, ten dimensional equations of motion allow for a class of solutions with $\mathcal{L}=0$, (3.53). The latter is equivalent to the relation (3.69). However, we can not impose (3.69) at the level of the potential $\mathcal{V}(3.65)$, so that

$$
\mathcal{V}(\sigma) \rightarrow \hat{\mathcal{V}}(\sigma)=\sigma^{-2} V_{-2}+\sigma^{4} V_{4}+V_{\text {sources }} .
$$

It is easy to see that though $\hat{\mathcal{V}}$ leads to the same value of the four dimensional cosmological constant, the equation of motion for the Kähler modulus $\sigma$ is inconsistent with ten dimensional equations of motion, unless $G=0$. Again, the problem appears because $\mathcal{V}(\sigma)$ is an off-shell quantity, and its partial evaluation on-shell, and subsequent

\footnotetext{
${ }^{10}$ In the supergravity approximation with localized D-branes sources.
} 
treatment of the result as off-shell quantity is inconsistent. From a slightly different angle, the inconsistency occurred because equations of motion for the fluxes depend on the overall size modulus $\sigma$ (a component of the ten dimensional metric), thus

$$
\left.\left(\left[\frac{d \mathcal{V}(\sigma)}{d \sigma}\right]^{\prime}\right)\right|_{E O M\{\text { fluxes }\}} \neq \frac{d}{d \sigma}\left[\left.\mathcal{V}(\sigma)\right|_{E O M\{\text { fluxes }\}}\right],
$$

since

$$
\left.\frac{d}{d \sigma}[\text { EOM fluxes }\}\right] \neq 0,
$$

where $E O M\{$ fluxes $\}$ stands for the fluxes equations of motion. Note, that it is not only on-shell fluxes that depend on the Kähler modulus. As explained in [21], the contribution to the four dimensional Kähler modulus effective potential from the $\overline{D 3}$ brane, $\delta V$, does not scale as $\sigma^{-3}$ as assumed in [8]. Rather, because the on-shell warp factor $e^{4 A} \sim \sigma$ [10], one finds (see Eq. (5.14) of [21])

$$
\begin{aligned}
\delta V & \sim \frac{1}{\sigma^{3}} e^{4 A} \sim \frac{1}{\sigma^{2}}, \\
& \equiv \frac{D}{(2 \sigma)^{2}} .
\end{aligned}
$$

Finally, in deriving the potential (3.65), we did not assume that the 3-from fluxes are ISD, i.e., $G^{+}=0$, or that $\tilde{\mathcal{M}}_{6}$ is Ricci flat, also we did not assume supersymmetry. Thus potential (3.65) can be useful to study effects of supersymmetry breaking and the backreaction of non-ISD fluxes in KKLT-like models.

\section{Acknowledgments}

I would like to thank Shanta de Alwis, Steve Giddings, Shamit Kachru, Renata Kallosh, Jim Liu, Rob Myers, Joe Polchinski, Radu Roiban, Christian Romelsberger, Sav Sethi, Henry Tye, and Johannes Walcher for numerous discussions, correspondence and comments. I would like to thank Aspen Center for Physics for hospitality where part of this work was done, and acknowledge the partial support by the U.S. Department of Energy while at the University of Michigan, where this work started. Research at the Perimeter Institute is supported in part by funds from NSERC of Canada. 


\section{Appendix}

Here we derive Eq. (3.57). First, multiply both sides of (3.40) by $e^{-4 A} \sqrt{\tilde{g}}$, and integrate over $\tilde{\mathcal{M}}_{6}$. Using definition (3.31), and the fact that

$$
\int_{\tilde{\mathcal{M}}_{6}} d^{6} y \sqrt{\tilde{g}} \tilde{\nabla}^{2} A=0
$$

we conclude

$$
\begin{aligned}
\Lambda= & -\frac{k_{4}^{2}}{2} \sum_{\text {sources }} T_{i} e^{4 A\left(y_{i}\right)}(\operatorname{Im} \rho)^{-3} \\
& -\frac{k_{4}^{2}}{k_{10}^{2}} \int_{\tilde{\mathcal{M}}_{6}} d^{6} y \sqrt{\tilde{g}}\left((\operatorname{Im} \rho)^{-3} \frac{1}{48} G_{m n p} \bar{G}^{m n p} e^{4 A}+(\operatorname{Im} \rho)^{4} 4(\tilde{\nabla} \omega)^{2} e^{-8 A}\right) .
\end{aligned}
$$

Let's multiply both sides of the Bianchi identity (3.42) by $e^{-8 A} \omega \sqrt{\tilde{g}}$ and integrate over $\tilde{\mathcal{M}}_{6}$. We find

$$
\begin{aligned}
& \frac{k_{4}^{2}}{k_{10}^{2}} \int_{\tilde{\mathcal{M}}_{6}} d^{6} y \sqrt{\tilde{g}}\left((\operatorname{Im} \rho)^{4} e^{-8 A} \omega\left(\tilde{\nabla}^{2} \omega-8 \tilde{\nabla} \omega \tilde{\nabla} A\right)-\frac{i}{48} \omega G \star_{6} \bar{G}\right) \\
& = \\
& -\frac{k_{4}^{2}}{2} \sum_{\text {sources }} q_{i}\left|T_{i}\right| \omega\left(y_{i}\right) .
\end{aligned}
$$

Given (3.85) and (3.86), Eq. (3.57) follows, provided

$$
\begin{aligned}
\mathcal{I} \equiv & 4(\operatorname{Im} \rho)^{4} e^{-8 A}\left(\omega \tilde{\nabla}^{2} \omega-8 \omega \tilde{\nabla} \omega \tilde{\nabla} A+(\tilde{\nabla} \omega)^{2}\right) \\
& +\left(-\frac{i}{12} \omega G \star_{6} \bar{G}+(\operatorname{Im} \rho)^{-3} \frac{1}{48} e^{4 A} G \bar{G}\right)
\end{aligned}
$$

integrates to zero over $\tilde{\mathcal{M}}_{6}$,

$$
\int_{\tilde{\mathcal{M}}_{6}} d^{6} y \sqrt{\tilde{g}} \mathcal{I}=0
$$

But (3.88) is trivial once we notice that

$$
\mathcal{I}=4(\operatorname{Im} \rho)^{4} \tilde{\nabla}\left(e^{-8 A} \omega \tilde{\nabla} \omega\right)-\frac{1}{48}(\operatorname{Im} \rho)^{-3} G \star_{6} \overline{\mathcal{L}}
$$

and recall that the 3-form Maxwell equation (3.49) implies (3.53). 


\section{References}

[1] G.W. Gibbons, "Aspects of supergravity theories" in Supersymmetry, Supergravity and Related Topics, edited by F. de Aguila, J.A. de Azcárraga and L. Ibañez, 346 (World Scientific, Singapore, 1985).

[2] J. M. Maldacena and C. Nunez, "Supergravity description of field theories on curved manifolds and a no go theorem," Int. J. Mod. Phys. A 16, 822 (2001) [arXiv:hep-th/0007018].

[3] A. Buchel, "Gauge / gravity correspondence in accelerating universe," Phys. Rev. D 65, 125015 (2002) [arXiv:hep-th/0203041].

[4] A. Buchel, P. Langfelder and J. Walcher, "On time-dependent backgrounds in supergravity and string theory," Phys. Rev. D 67, 024011 (2003) [arXiv:hepth/0207214].

[5] A. Buchel, "Compactifications of the $\mathrm{N}=2^{*}$ flow," Phys. Lett. B 570, 89 (2003) [arXiv:hep-th/0302107].

[6] P. K. Townsend and M. N. Wohlfarth, "Accelerating cosmologies from compactification," Phys. Rev. Lett. 91, 061302 (2003) [arXiv:hep-th/0303097].

[7] S. P. de Alwis, "On potentials from fluxes," arXiv:hep-th/0307084

[8] S. Kachru, R. Kallosh, A. Linde and S. P. Trivedi, "De Sitter vacua in string theory," Phys. Rev. D 68, 046005 (2003) [arXiv:hep-th/0301240].

[9] S. Gukov, C. Vafa and E. Witten, "CFT's from Calabi-Yau four-folds," Nucl. Phys. B 584, 69 (2000) [Erratum-ibid. B 608, 477 (2001)] [arXiv:hep-th/9906070].

[10] S. B. Giddings, S. Kachru and J. Polchinski, "Hierarchies from fluxes in string compactifications," Phys. Rev. D 66, 106006 (2002) [arXiv:hep-th/0105097].

[11] E. Cremmer, S. Ferrara, C. Kounnas and D. V. Nanopoulos, "Naturally Vanishing Cosmological Constant In N=1 Supergravity," Phys. Lett. B 133, 61 (1983). J. R. Ellis, A. B. Lahanas, D. V. Nanopoulos and K. Tamvakis, "No - Scale Supersymmetric Standard Model," Phys. Lett. B 134, 429 (1984). 
[12] E. Witten, "Dimensional Reduction Of Superstring Models," Phys. Lett. B 155, 151 (1985).

[13] M. Dine, R. Rohm, N. Seiberg and E. Witten, "Gluino Condensation In Superstring Models," Phys. Lett. B 156, 55 (1985).

[14] E. Witten, "Non-Perturbative Superpotentials In String Theory," Nucl. Phys. B 474, 343 (1996) [arXiv:hep-th/9604030].

[15] S. B. Giddings, "The fate of four dimensions," Phys. Rev. D 68, 026006 (2003) [arXiv:hep-th/0303031].

[16] A. Buchel, talk at 3rd International Symposium on Quantum Theory and Symmetries, Cincinnati, 2003.

[17] O. DeWolfe and S. B. Giddings, "Scales and hierarchies in warped compactifications and brane worlds," Phys. Rev. D 67, 066008 (2003) [arXiv:hep-th/0208123].

[18] J. H. Schwarz, "Covariant Field Equations of Chiral $N=2 D=10$ Supergravity," Nucl. Phys. B226 (1983) 269.

[19] A. Buchel, A. W. Peet and J. Polchinski, "Gauge dual and noncommutative extension of an $N=2$ supergravity solution," Phys. Rev. D 63, 044009 (2001) [arXiv:hep-th/0008076].

[20] A. R. Frey, M. Lippert and B. Williams, "The fall of stringy de Sitter," Phys. Rev. D 68, 046008 (2003) [arXiv:hep-th/0305018].

[21] S. Kachru, R. Kallosh, A. Linde, J. Maldacena, L. McAllister and S. P. Trivedi, "Towards inflation in string theory," JCAP 0310, 013 (2003) [arXiv:hepth/0308055]. 